\title{
Parasitoid and host nutritional physiology in behavioral ecology
}

\author{
Michael R. Strand and Jérôme Casas
}

\begin{abstract}
Nutrient acquisition and allocation critically impacts the fitness of all organisms. However, distinguishing between competing hypotheses about metabolic strategies requires mechanistic details on the physiological requirements and constraints under which the organism exists. Parasitoids acquire nutrients during both their larval stage and as adults. In this chapter, we first explore differences in the acquisition strategies of koinobionts and idiobionts and the physiological adaptations parasitoids have evolved to manipulate host nutrient stores for their own fitness. We then relate these larval strategies to nutrient acquisition strategies during the adult stage by revisiting the link between reproduction (ovigeny index), host-feeding behavior, and oosorption. We conclude our discussion by examining how nutritional interactions potentially impact on other aspects of parasitoid and host fitness. Overall, we argue that the combination of top-down evolutionary approaches with bottom-up insights into physiology and molecular mechanisms offers new avenues for understanding the complex syndromes of the idiobiont and koinobiont life history dichotomy.
\end{abstract}

\subsection{Introduction}

Classic life history models assume that fundamental trade-offs will arise between reproduction and determinants of survival when resources are limiting. Many factors can constrain resource availability including ecological conditions and the structure of an organism's life cycle. Parasitoids acquire nutrients as larvae by feeding on hosts (capital resources). They can also acquire additional nutrients as adults by feeding on non-host resources like nectar and/or by host feeding (income resources) (Jervis \& Kidd 1986, Heimpel \& Collier 1996). Most behavioral and population dynamics models measure the quality of host resources 
using a single currency such as size (Charnov \& Skinner 1984, Mangel 1989). Parasitoid nutrient reserves are also represented usually by single currencies like the number of eggs available for oviposition (egg load) to predict parasitoid foraging decisions or populationlevel effects on hosts (Mangel 1989, Chan \& Godfray 1993, Briggs et al. 1995, Collier 1995, Krrivan 1997, Heimpel et al. 1998, Rosenheim et al. 2000). In reality, nutrient acquisition and allocation is much more complex, because individual nutrients can be limiting for specific functions, such as reproduction, even when energetic resources are not (Raubenheimer \& Simpson 1999, O'Brien et al. 2002). Changes in diet or metabolism can also affect how nutrients from different life stages are used (Zera \& Zhao 2003, O’Brien et al. 2004, Min et al. 2006).

Hosts face similar complexities in nutrient acquisition and allocation. They also face potentially important fitness trade-offs from investing in defense against attack by parasitoids and pathogens relative to reproduction and other maintenance needs (see Chapter 14 by Kraaijeveld and Godfray). Defense against internal parasitoids depends primarily on the ability of the host's immune system to kill the parasitoid egg or larva after oviposition (Strand \& Pech 1995, Lavine \& Strand 2002, Hoffmann 2003, see also Chapter 14 by Kraaijeveld and Godfray). Host-parasitoid population dynamics are also affected by variation in host resistance, which can arise as a consequence of genetic differences among individuals or from environmental factors such as nutritional state (Chesson \& Murdoch 1986, Hochberg 1997, Sasaki \& Godfray 1999, Godfray 2000, Carton et al. 2005).

In this chapter we examine the effects of nutritional physiology on the behavioral ecology of parasitoids and their hosts. We begin by outlining the relationship between life history and nutrient dynamics in parasitoids. We then explore the role of nutritional state in immunity and the potential costs of defense to hosts (see also Chapter 14 by Kraaijeveld and Godfray). We finish by arguing that the combination of top-down ecological, population, and evolutionary studies combined with bottom-up insights from physiology and molecular biology offers new avenues for understanding the behavioral ecology of parasitoids.

\subsection{Background nutritional physiology}

Carbohydrates, proteins, and lipids are the primary nutrient classes and the fat body is the main site of nutrient storage and metabolism for all insects. The fat body stores carbohydrates as glycogen and lipids as tryglycerides (Clements 1992, Candy et al. 1997). The fat body also synthesizes many key molecules including the lipoprotein vitellogenin, the primary constituent of yolk, and trehalose, which is a key sugar in hemolymph. Insect hemolymph is another site of nutrient storage and usually contains high levels of free amino acids, storage proteins, and sugars required for maintenance, metamorphosis, and reproduction. Regulation of nutrient homeostasis (i.e. nutrient sensing) in insects, as in vertebrates, occurs primarily through the insulin and the target of rapamycin (TOR) pathways (Britton et al. 2002, Scott et al. 2004). Insulin signaling is a hormone-based system that regulates metabolism and organismal growth while the TOR pathway responds to nutrient levels to regulate protein synthesis and cell growth (Oldham et al. 2000, Zhang et al. 2000, Wu \& Brown 2006). Reciprocally, inhibition of the TOR pathway during starvation induces autophagy, whereby non-essential proteins and organelles are recycled to generate amino acids for other purposes (Scott et al. 2004). 


\subsection{Nutrient acquisition and allocation by parasitoids varies with life history}

Nutrient acquisition and allocation strategies of parasitoids are strongly linked to two components of life-history: (i) egg production and (ii) mode of parasitism. For the former, Flanders (1950) divided parasitoids into pro-ovigenic species that emerge as adults with a fixed complement of mature eggs and synovigenic species that continue to mature eggs during the adult stage. Pro-ovigenic parasitoids allocate nutrient reserves during the adult stage to maintenance, while synovigenic parasitoids confront the decision of whether to allocate reserves to egg production, maintenance, or both (Jervis \& Kidd 1986, Heimpel \& Collier 1996, Rivero \& Casas 1999, Papaj 2000). Jervis et al. (2001) noted that parasitoids actually exhibit a continuum of ovigeny that can be indexed. Relatively few species are strictly pro-ovigenic (ovigeny index $=1$ ) and synovigeny ranges from species that emerge with most eggs mature to species that emerge with no mature eggs (ovigeny index $=0$ ). Askew and Shaw (1986), in contrast, divided parasitoids into idiobionts, whose hosts cease development after parasitism and koinobionts, whose hosts remain mobile and continue to grow. All idiobionts are either ectoparasitoids that paralyze their hosts or endoparasitoids that parasitize sessile host stages like eggs or pupae. Most koinobionts in contrast are endoparasitoids that parasitize insect larvae.

Egg production and mode of parasitism strategies are also interrelated. Parasitoids that exhibit extreme synovigeny, for example, are all idiobionts that produce yolk-rich (anhydropic) eggs and low ovigenic indices, whereas koinobionts tend to produce yolkdeficient (hydropic) eggs and have high ovigenic indices (Mayhew \& Blackburn 1999). Since idiobionts parasitize hosts of static size, selection favors oviposition on larger, late-stage hosts, which suffer lower mortality rates and thus select for larger egg sizes and concomitantly lower fecundities (Price 1974, Mackauer \& Sequeira 1993, Godfray 1994). Most idiobionts are also ectoparasitoids whose eggs require a pre-packaged yolk source for development. Reciprocally, the ability of koinobionts to attack hosts of variable size favors parasitism of early stage larval hosts that suffer higher mortality rates and thus, favor smaller eggs and larger fecundities. The production of smaller, yolk-deficient eggs is also probably favored in koinobionts by: (i) endoparasitism and access to host nutrients for embryonic development; and (ii) the inability of koinobionts to feed on the mobile, often aggressive, hosts they attack (Strand 2000, Pennacchio \& Strand 2006).

\subsection{Parasitoid nutrient dynamics}

With these life history correlations in mind, it is not surprising that most idiobionts are able to lay a few eggs after adult emergence, but further increases in longevity and egg production require host feeding and/or access to non-host resources like nectar (Jervis \& Kidd 1986, Heimpel \& Collier 1996, Rivero \& Casas 1999). Using biochemical methods and dietary stable isotope signatures, more recent studies have also begun to unravel the contribution of larval and adult diets to parasitoid nutrient budgets. Nutrients that are acquired during the larval stage and used during the adult stage are referred to as capital reserves. Studies of the ectoparasitic idiobiont Eupelmus vuilleti indicate that adult females emerge with high capital reserves of lipid, sugars, and glycogen. Host and sugar feeding 
by adults provides additional protein and carbohydrates but little or none of these nutrients are converted to lipid suggesting an absence of lipogenesis during the adult stage of E. vuilleti (Giron \& Casas 2003). Lipid ingested by host feeding is also inadequate to replace capital lipid reserves from the larval stage (Giron et al. 2002, Casas et al. 2005). In contrast, the endoparasitic koinobiont Venturia canescens is weakly synovigenic, produces small eggs with little yolk, and does not host feed. This species emerges with limited capital reserves, is stored mainly as lipids, and possesses almost no reserves stored in eggs themselves (Casas et al. 2003). Carbohydrate levels increase rapidly in V. canescens from feeding on nectar and/or honeydew, yet, similar to E. vuilleti, lipid reserves do not (Casas et al. 2003).

Strong synovigeny has previously been associated with weak capital reserves compared to weak synovigeny or pro-ovigeny (Jervis et al. 2001), yet the preceding data suggest this is not the case. Instead, nutrient reserves must be viewed in terms of individual nutrient classes (Section 6.6). Carbohydrates, for example, obtained by adults from host or nonhost sources enhance longevity and survival of both idio- and koinobionts. Carbohydrates also appear to be the only nutrient used for flight by Hymenoptera and this nutrient class is rapidly depleted if adults are starved (Vogt et al. 2000, Harrison \& Fewell 2002). Proteins and carbohydrates obtained by idiobionts through host feeding increase the production of yolk-rich eggs by allowing females to invest less capital lipid reserves in maintenance functions. In contrast, neither E. vuilleti nor $V$. canescens is able to synthesize new lipids from carbohydrates as adults, making lipids acquired during larval development a non-renewable resource that ultimately constrains egg production (see below). Comparative studies have further suggested an absence of lipogenesis during the adult stage may exist across all Hymenoptera (Ellers 1996, Rivero \& Casas 1999, Olson et al. 2000, Rivero et al. 2001, Rivero \& West 2002). If so, we would hypothesize that constraints on lipid reserves are a major factor influencing the foraging behavior and oviposition strategies of parasitoid wasps.

Given that koinobionts are unable to host feed, we would also suggest that many species have evolved compensatory strategies for enhancing the acquisition of nutrients during larval development that are lacking in the adult diet. This would include lipids, sterols, and non-essential amino acids that are deficient in nutrient sources available to adults like nectar (O’Brien et al. 2002, 2004). Notably, many larval endoparasitoids induce dramatic reductions in weight gain and inhibit metamorphosis by hosts (Harvey \& Strand 2002, Beckage \& Gelman 2004, Pennacchio \& Strand 2006). These developmental alterations are also associated with qualitative and quantitative alterations in how carbohydrates, proteins, and lipids are allocated to host tissues (Thompson 1993, Thompson \& Dahlman 1998, Pennacchio \& Strand 2006). Key changes include large increases in host hemolymph carbohydrate levels and alterations in protein composition including a loss of major storage proteins like arylphorin (Dahlman \& Vinson 1980, Thompson 1982, Vinson 1990, Shelby \& Webb 1994). Reductions in triglyceride and glycogen deposits in fat body and increases in hemolymph protein, amino acid, and acyl-glycerol levels have also been reported in aphids parasitized by aphidiine braconids (Pennacchio et al. 1995, Rahbé et al. 2002). Although idiobionts have traditionally been thought to manipulate host physiology less than koinobionts (Askew \& Shaw 1986, Jervis et al. 2001), recent studies have suggested this generality may also be inaccurate (Pennacchio \& Strand 2006). For example, the venoms produced by idiobionts are well known for their paralytic activity. However, idiobiont venoms also cause several endocrine and metabolic alterations that increase 
carbohydrate, protein, and lipid levels in host hemolymph that is the primary resource consumed by developing parasitoid larvae (Strand 1986, Rivers \& Denlinger 1994, Weaver et al. 2001).

The molecular mechanism(s) underlying these metabolic alterations remain only superficially understood (see Thompson 1993, Beckage \& Gelman 2004, Pennacchio \& Strand 2006). Nonetheless, Pennacchio and Strand (2006) concluded that their overall effect is to redirect energetic resources away from the host and toward the developing larval stage parasitoid so as to enhance capital reserves. As discussed above, koinobionts almost always juvenilize their larval stage hosts in a manner that increases nutrient availability in the hemolymph and disrupts nutrient uptake by host tissues. Parasitoids that attack nymphal or adult hosts confront a very different host environment but the strategy of manipulating host nutritional physiology remains similar. This is well illustrated by aphid parasitoids like Aphidius ervi. The primary metabolic sink of its host is reproduction and, not surprisingly, A. ervi produces gene products that suppress host reproduction (Pennacchio et al. 1995, Digilio et al. 2000). Thus, the primary adaptive significance of altering host endocrine physiology and reproduction is likely metabolic with arrested development or inhibition of metamorphosis being indirect consequences of the parasitoid redirecting host nutritional resources. Redirecting nutrient reserves away from the host and toward the parasitoid also potentially reduces immune defenses, which could further favor parasitoid survival (see below).

\subsection{Nutrient dynamics and immune defense by hosts}

Host insects similarly confront resource constraints that can affect their life history. In Lepidoptera, for example, sugar feeding by adults increases longevity and fecundity, but essential amino acids are acquired primarily from the larval diet, which places an upper limit on the use of adult dietary resources for reproduction (O'Brien et al. 2002, 2004). Even in host insects with similar larval and adult diets, resource allocation of nutrients acquired during the larval and adult phase can vary with time or by tissue. Dietary sucrose and yeast provides virtually all of the carbon Drosophila melanogaster allocates to eggs, but the origin of these sugar carbons shifts from larval sources in the first clutches of eggs laid by a female to almost exclusively adult sources in subsequent clutches. In contrast, more than $30 \%$ of sugar carbons allocated to adult somatic tissues derive from larval reserves, suggesting that resources acquired during different life stages are allocated differently to maintenance and reproduction (Min et al. 2006).

Since parasitoids and pathogens are often the most important mortality factors facing insects, immune defense has long been recognized as a critically important maintenance function (see Chapter 14 by Kraaijeveld and Godfray). Investment in defense also has possible fitness costs and trade-offs with reproduction and other needs. Such trade-offs have been viewed as either a plastic response, usually referred to as the cost of using the immune system or as a co-evolved trait viewed as the cost of having an immune system (Schmid-Hempel \& Ebert 2003, see also Chapter 14 by Kraaijeveld and Godfray). The innate immune response of insects consists of both cellular and humoral components (Strand \& Pech 1995, Lavine \& Strand 2002, Hoffmann 2003). Some defenses, like melanization, are non-specific and have activity against a range of parasites, while other defenses are directed to a restricted set of parasite species or types. The primary defense response against 
parasitoids is encapsulation, which involves the recognition and binding of hemocytes to the parasitoid egg or larva. Binding of additional hemocytes ultimately results in a multicellular capsule that fully envelops the parasitoid (see Chapter 14 by Kraaijeveld and Godfray). The encapsulated parasitoid is then likely to be killed by a combination of asphyxiation and toxic compounds produced by the melanization pathway that hemocytes often release in mature capsules (Wertheim et al. 2005). Several immune pathways regulate encapsulation and associated processes such as hemocyte proliferation and activation of the phenoloxidase cascade, which regulates melanization (Irving et al. 2005, Wertheim et al. 2005, see Chapter 14 by Kraaijeveld and Godfray). These include the Toll and immune deficiency (Imd) pathways that also regulate defense responses to microbial infection and the JAK-STAT (Janus kinase and signal transducers) and GATA signaling pathways that regulate hematopoiesis and antiviral defense (Hoffmann 2003, Agaisse \& Perrimon 2004). These pathways and the effector responses they regulate together comprise the insect's inflammatory response to infection.

\subsubsection{Is immune defense costly?}

If there are life history costs associated with resistance, then the benefit to hosts of investing in immune defense will be determined by the risk of attack, which will vary in both space and time (see also Chapter 14 by Kraaijeveld and Godfray). Resistance would also be expected to vary within and between host populations and could be affected by genetic as well as environmental factors. There is considerable evidence for host variation in immune resistance to parasitoid attack both between (Kraaijeveld et al. 1998, Hufbauer 2002, Carton et al. 2005) and within (Henter \& Via 1995, Kraaijeveld \& Godfray 1997, Fellowes 1999, Kraaijeveld \& Godfray 1999, Stacey \& Fellowes 2002, Gwynn et al. 2005) host populations (see Chapter 14 by Kraaijeveld and Godfray). A few recent studies have also suggested that immune resistance to parasitoids has fitness costs for survival and future reproduction. For example, selection for increased immune resistance in $D$. melanogaster to the parasitoids Asabara tabida and Leptoplinina boulardi results in enhanced encapsulation of parasitoids, but at the cost of a reduction in the ability of resistant hosts to compete for food (Kraaijeveld \& Godfray 1997, Fellowes et al. 1999, see also Chapter 14 by Kraaijeveld and Godfray). Aphids usually do not eliminate parasitoids by encapsulation but other immune factors in hemolymph prevent parasitoids eggs from developing. This immune response also appears to have a cost, because pea aphid clones (Acyrthosiphon pisum) with higher immune resistance to the parasitoid Aphidium ervi have lower fecundity than susceptible clones (Gwynn et al. 2005). Outside of the parasitoid literature, other evidence for the cost of immune defense includes reductions in survival and male mating success (Rolff \& Siva-Jothy 2002, Schmid-Hempel \& Ebert 2003). While the examples cited here provide evidence of trade-offs between immunity, reproduction, and/or other maintenance functions, additional comparative data and experimentation are also needed to determine whether the costs of immune defenses are broadly significant among different host-parasitoid associations.

\subsubsection{Is resistance affected by nutrient dynamics?}

Evidence that immune defense is energetically expensive in insects and other organisms derives from the observation, in diverse species, that activation of the immune response 
increases mortality or has other measurable fitness consequences when resources are limited (Rolff \& Siva-Jothy 2003, Schmidt-Hempel \& Ebert 2003). The prevalence of induced versus constitutive responses in immunity following parasite infection is also thought to have evolved, in part, to avoid the energetic cost of permanent defense. Support for this latter idea derives primarily from genetic modification of immune traits or selection studies. For example, mutations that constitutively activate systemic acquired resistance in plants increase resistance to certain pathogens but at the cost of reduced size and seed production (Heidel et al. 2004). Persistent activation of the pathways regulating antimicrobial peptide production in $D$. melanogaster also reduces fecundity presumably due to resources invested in defense molecules being unavailable for egg production (Zerofsky et al. 2005). As mentioned above, D. melanogaster selected for resistance to parasitoid attack produce more hemocytes but at the cost of reduced competitive ability under conditions of high resource competition (Kraaijeveld \& Godfray 1997, Kraaijeveld et al. 1998). Surprisingly though, flies selected for increased competitive ability under crowding do not exhibit reduced resistance to parasitoids (Sanders et al. 2005).

Other evidence for the energetic cost of immune defense stems from the observation that stress, including high temperature, starvation, or persistent infection, increases susceptibility to infection due to a presumed reduction in resources available for immunity (Boulétreau 1986, Feder et al. 1997, Faggioni et al. 2000, Yang \& Cox-Foster 2005). Insect resistance to parasitoids can also vary by age with younger larvae or older adults usually being most susceptible to infection (Salt 1970, Washburn et al. 2001, Hillyer et al. 2005, Zerofsky et al. 2005). This increased susceptibility could be due to age-dependent changes in the function of the immune system, lower nutrient reserves for investment in defense, or both.

Outside of insects, several linkages have also been identified between metabolic and immune pathways (Lochmiller \& Deerenberg 2000, Rolff \& Siva-Jothy 2003, Matarese \& La Cava 2004). Pro-inflammatory cytokines and persistent infection by pathogens, such as tuberculosis, triggers hyperglycemia in mammals due to insulin insensitivity and release of glucocorticoids (Andersen et al. 2004). This shift in nutrient allocation affects both immune cell function and the production of acute phase proteins, but at the cost of reduced skeletal muscle mass and impaired glycogen synthesis. As noted above, parasitoids induce a similar response in host insects (Pennacchio \& Strand 2006). Starvation in insects is also known to alter both TOR and immune pathway signaling (Gordon et al. 2005).

Lastly, a few studies have documented genetic differences in metabolic enzymes that have distinct pleiotropic effects in different life stages that could result in trade-offs between defense, reproduction, and other maintenance functions. For example, the glycolytic enzyme phosphoglucose isomerase (Pgi) plays a key role in glucose metabolism and the resupply of energy (ATP). Studies in several insects indicate that different Pgi genotypes exhibit variation in their enzyme kinetic and thermal stability properties that correlate with variation in flight performance, adaptation to particular microhabitats, and fecundity (Watt 1992, Hanski \& Saccheri 2006, Giron et al. 2007). Lepidotopteran larvae feeding on diets with high concentrations of plant toxins have also been reported to suffer higher rates of parasitism due potentially to the energetic costs of detoxification reducing resources available for immune defense (Gentry \& Dyer 2002). In contrast, other studies have found little or no evidence that processing of allelochemicals imposes significant energy demands on insect herbivores, making it unclear whether increased vulnerability to parasitoids is actually due to metabolic trade-offs (Appel \& Martin 1992). 


\subsection{The power of a complete nutrient budget}

As shown above, nutrient acquisition and allocation clearly impact on fecundity and survival functions such as immune defense in both parasitoids and hosts. Yet, these linkages are also founded more on logical deductions than hard data, indicating that this field of study would strongly benefit from additional experimental data. The deficiencies in available empirical data in specific host-parasitoid study systems is also well illustrated in the theoretical literature by the types of dynamic models that have been developed to predict the behavior and host usage patterns of parasitoids. These models are often very detailed and strive for realism. However, resolving differences in the predictions generated by different models has also been noted to be 'essentially impossible', because of a lack of physiological knowledge about nutrient dynamics in parasitoids (Clark \& Mangel 2000). Similarly, while several lines of evidence suggest immune defense is energetically expensive to hosts, the precise trade-offs between defense, reproduction, and other fitness traits remain largely undefined.

To address these challenges, we conclude that quantified energy and nutrient budgets for both parasitoids and hosts are the only way to make sense of the myriad of evolutionary scenarios that can arise. The key advantage to this approach is that it enables us to assess the relative benefits and costs of different nutrient acquisition and allocation strategies. Building complete budgets is not an easy task but it is doable, even in small parasitoids. This is illustrated by the comprehensive total energy budget recently developed for the idiobiont Eupelmus vulleti that we discussed earlier in this chapter (Casas et al. 2005, see above). For this analysis, the sugar, glycogen, protein, and lipid reserves of single females at birth and death were quantified, as was daily maintenance. Each host feeding and oviposition event, along with the nutrient amounts acquired and invested in eggs, was recorded. The time of death was also used to compare model predictions in the presence and absence of hosts. In the absence of host feeding, nutrients derive from larval (capital) reserves. The availability of host feeding, on the other hand, delivers large quantities of sugars and proteins but very little lipid (Fig. 6.1). In terms of allocation, carbohydrates are the main energy source for maintenance functions and the decline in carbohydrate reserves mirrors the time of death. When adults can host feed but have no supplemental sugar source, proteins and lipids are heavily used for maintenance, which in turn allows females to use capital lipid reserves acquired during larval feeding for egg production (Fig. 6.2). Providing adults both sugar and hosts increases longevity, reduces host feeding, and produces higher realized fecundities because most capital lipid reserves are used for egg production (Fig. 6.2).

Overall, these data indicate that $E$. vuilleti is best described as a capital breeder for one nutrient class (lipids) and is an income breeder for another (carbohydrates). The nutrient budget developed for this species also illustrates the inadequacy of a term such as 'energy' for describing nutrient reserves and the importance of recognizing that different nutrient classes are not used equivalently for reproduction and maintenance. Even categories such as protein, lipid, and carbohydrate are potentially too coarse, as illustrated recently by Mondy et al. (2006), who found that E. vuilleti is actually an income breeder for at least one subclass of lipids (sterols), because capital sterol reserves appear sufficient to produce only $30 \%$ of the total number of eggs females normally lay when given access to hosts and sugar. 
Nutrient acquisition without host feeding

Lipids

Carbohydrates

Proteins

Fig. 6.1 Lifetime acquisition and allocation of nutrients in Eupelmus vuilleti when adults are without or with hosts for host feeding. Larval reserves of lipid, carbohydrate, and proteins are indicated in black. Nutrients gained through host feeding are indicated in white. When wasps are able to host feed, most lipid reserves still derive from the larval stage, whereas the majority of carbohyd rates and proteins are acquired from host feeding. This indicates that $E$. vuilleti is a capital breeder in terms of lipids, but is an income breeder in terms of carbohydrates and protein. Allocation of lipids, carbohydrates, and proteins to maintenance functions is indicated in light gray, while allocation to eggs is indicated in dark gray.
Nutrient acquisition
Nutrient acquisition with host feeding
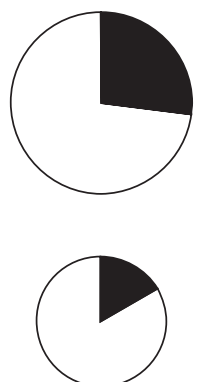

Nutrient allocation with host feeding
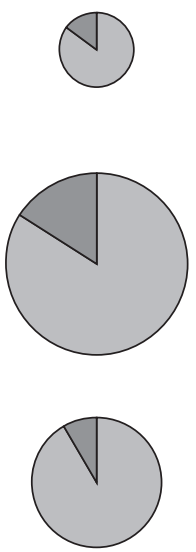

Nutrient

allocation

Lipids

\section{Without}

sugar
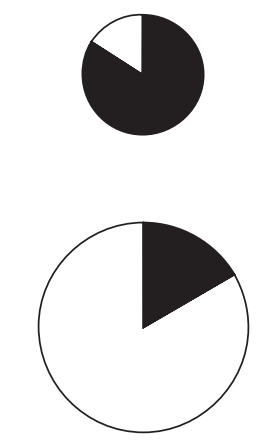
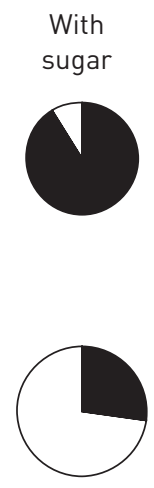

Proteins

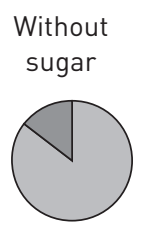

With sugar

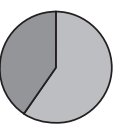

Fig. 6.2 Lifetime acquisition and allocation of nutrients in Eupelmus vuilleti when adults can host feed with or without supplemental sugars. Note that acquisition of additional carbohydrates during the adult stage enables females to increase the allocation of lipids and protein to egg production. Sugar acquisition also reduces host-feeding, which results in a decrease in protein reserves. 
The development of complete nutrient budgets can also be used to tackle difficult questions about the relative costs and benefits of particular physiological functions and behaviors. For example, egg resorption is a common phenomenon in parasitoids and other insects that is usually explained as a strategy for recovering nutrients under conditions of resource limitation and reallocating them to maintenance (Bell \& Bohm 1975). A few studies with parasitoids have reported a positive correlation between the number of eggs resorbed and extended longevity (Collier 1995, Heimpel et al. 1997). Yet, quantitative data with $E$. vuilleti indicate that single eggs contain less than $10 \%$ of the protein, lipid, and carbohydrate needed for daily maintenance, suggesting a female cannot extend its longevity even a full day by reabsorption of its entire egg complement. Thus, egg reabsorption is either of value under only the most extreme conditions of resource limitation or it is important for other, currently unrecognized, functions such as oocyte survival. For hosts, a complete nutrient budget could also provide important insights into the actual cost of immune defense against particular parasitoids and trade-offs between processing allelochemicals and defense against parasitoids. The role of different nutrient classes in the metabolic costs of the immune system is only beginning to be characterized in mammals (Lochmiller \& Deerenberg 2000) and is almost completely unknown in insects.

\subsection{Nutrient dynamics and the future of behavioral and population ecology}

The last decade has produced a wave of exciting new data on nutrient dynamics of parasitoids and some hosts, but we are not yet at a stage where a quantitative fitness gain can be ascribed to a single nutrient acquisition or allocation decision. Nonetheless, such data are now possible to generate and are likely to enter the literature in the near future. It is at the population level that the state of the art is more worrisome. Demanding quantitative tools on one hand and a general decrease in the number of researchers studying population dynamics on the other is reducing progress in this area, despite its broadly acknowledged importance to the study of parasitoid-host relationships. Increased emphasis on physiological realism has pervaded host-parasitoid population dynamics models during the last decade but predictions about the stability of these interactions still require details on nutrient acquisition and allocation that are not available in most study systems. For example, recent models predict that allocation of nutrients gained from host feeding to both maintenance and reproduction will result in destabilizing population dynamics (Kidd \& Jervis 1991a,b, Briggs et al. 1995, Křivan 1997). Models also predict destabilizing dynamics if eggs are reabsorbed for maintenance purposes (Briggs et al. 1995). In contrast, if the parasitoid death rate is a function of nutrient reserves, then a failure to meet maintenance requirements is predicted to result in stabilizing dynamics. These models, therefore, predict that parasitoid-host systems can shift from population stability to instability depending on how nutrients acquired by host feeding are allocated and the relationship between death rate and reserves. To date, the physiologically structured host-parasitoid population models of Murdoch et al. (2003) provide the most thorough groundwork on the interface between physiology and population dynamics. Unfortunately, parasitoids, like Aphytis sp., for which we have a good grasp of population dynamics, are poorly understood from the perspective of nutritional physiology. In other words, inadequate detail about the nutritional physiology of these wasps precludes different model predictions from 
being thoroughly tested. In addition, while resolving questions of stability and instability are the bred and butter of population dynamicists, these issues are usually of little interest to physiologists and molecular biologists. The challenge then is how to engage these suborganismal biologists, who can generate quantitative measures of specific nutrient classes and their trafficking, on the importance of producing data that could help distinguish between competing model predictions that lead to opposite dynamic outcomes. This challenge also holds in the study of host immune resistance and parasitoid virulence, where understanding of how variation in non-genetic factors, like nutritional state or the coevolutionary dynamics that maintain genetic variation requires details on the physiological processes involved.

Behavioral and population ecologists would argue that the best route to deciding which physiological processes are most relevant to the foraging and population biology of a parasitoid would be to work in a top-down fashion using dynamic programming models for behavioral decisions or physiologically structured models for population dynamics. Model exploration and sensitivity analysis enable one to identify the relevant processes, i.e. those for which a small change in parameter values or function has a major impact. This can then be followed by experimental studies that generate the necessary physiological data for testing model predictions. Physiologists and molecular biologists, on the other hand, would point to the power and value of bottom-up approaches of characterizing genetic mutants, conducting selection studies, and undertaking functional genomic analyses to identify genes of interest and to understand how variation in specific traits impacts on function. The truth is that both strategies have value in enhancing our understanding of parasitoid-host interactions. This chapter even provides evidence that physiologists/ molecular biologists and ecologists/population biologists are capable of working together toward a common goal. We hope that the next decade will experience a move from plausible logical arguments about fitness gains under different ecological scenarios to quantitative predictions matched by experimental data of equivalent precision.

\section{Acknowledgments}

We thank Éric Wajnberg and three anonymous referees for valuable suggestions on an earlier draft of this manuscript. Some aspects of the work discussed in this chapter were supported by grants from the National Science Foundation, US Department of Agriculture, and National Institutes of Health to Michael R. Strand.

\section{References}

Agaisse, H. and Perrimon, N. (2004) The role of JAK/STAT signalling in the Drosophila immune response. Immunology Reviews 198: 72-82.

Andersen, S.K., Gjedsted, J., Christiansen, C. and Tonnesen, E. (2004) The roles of insulin and hyperglycemia in sepsis pathogenesis. Journal of Leukocyte Biology 75: 413-21.

Appel, H.M. and Martin, M.M. (1992) Significance of metabolic load in the evolution of host specificity of Manduca sexta. Ecology 73: 216-28.

Askew, R.R. and Shaw, M.R. (1986) Parasitoid communities. Their size, structure and development. In: Waage, J.K. and Greathead, D. (eds.) Insect Parasitoids. Academic Press, London, pp. 225-64. 
Beckage, N.E. and Gelman, D.B. (2004) Wasp parasitoid disruption of host development: implications for new biologically based strategies for insect control. Annual Review of Entomology 49: 299-330.

Bell, W.J. and Bohm, M.K. (1975) Oosorption in insects. Biological Review 50: 373-96.

Boulétreau, M. (1986) The genetic and coevolutionary interactions between parasitoids and their hosts. In: Waage, J.K. and Greathead, D. (eds.) Insect Parasitoids. Academic Press, London, pp. 169-200.

Briggs, C.L., Nisbet, R.M., Murdoch, W.W., Collier, T.R. and Metz, J.A.J. (1995) Dynamical effects of host-feeding in parasitoids. Journal of Animal Ecology 64: 403-16.

Britton, J.S., Lockwood, W.K., Li, L., Cohen, S.M. and Edgar, B.A. (2002) Drosophila's insulin/PI3kinase pathway coordinates cellular metabolism with nutritional conditions. Developmental Cell 2: 239-49.

Candy, D.J., Becker, A. and Wegener, G. (1997) Coordination and integration of metabolism in insect flight. Comparative Biochemistry \& Physiology B 117: 497-512.

Carton, Y., Nappi, A. and Poirié, M. (2005) Genetics of anti-parasite resistance in invertebrates. Developmental \& Comparative Immunology 29: 9-32.

Casas, J., Driessen, G., Mandon, N. et al. (2003) Strategies of energy acquisition and use of a parasitoid in the wild. Journal of Animal Ecology 69: 691-7.

Casas, J., Pincebourde, S., Mandon, N., Vannier, F., Poujol, R. and Giron, D. (2005) Lifetime nutrient dynamics reveal simultaneous capital and income breeding in a parasitoid. Ecology 86: 545-54.

Chan, M.S. and Godfray, H.C.J. (1993) Host-feeding strategies of parasitoid wasps. Evolution 7: 593-604.

Charnov, E.L. and Skinner, S.W. (1984) Evolution of host selection and clutch size in parasitoid wasps. Florida Entomologist 67: 5-21.

Chesson, P.L. and Murdoch, W.W. (1986) Aggregation of risk: relationships among host-parasitoid models. American Naturalist 127: 696-715.

Clark, C.W. and Mangel, M. (2000) Dynamic State Variable Models in Ecology. Oxford University Press, New York.

Clements, A.N. (1992) The Biology of Mosquitoes, vol. I. Chapman \& Hall, London.

Collier, T.R. (1995) Adding physiological realism to dynamic state variable models of parasitoid hostfeeding. Evolutionary Ecology 9: 217-35.

Dahlman, D.A. and Vinson, S.B. (1980) Glycogen content in Heliothis virescens parasitized by Microplitis croceipes. Comparative Biochemistry \& Physiology A 66: 625-30.

Digilio, M.C., Isidoro, N., Tremblay, E. and Pennacchio, F. (2000) Host castration by Aphidius ervi venom proteins. Journal of Insect Physiology 46: 1041-50.

Ellers, J. (1996) Fat and eggs: an alternative method to measure the trade-off between survival and reproduction in insect parasitoids. Netherlands Journal of Zoology 46: 227-35.

Faggioni, R., Moser, A., Feingold, K.R. and Grunfeld, C. (2000) Reduced leptin levels in starvation increase susceptibility to toxic shock. American Journal of Pathology 156: 1781-7.

Feder, D., Mello, C.B., Garcia, E.S. and Azambuja, P. (1997) Immune responses in Rhodnius prolixus: influence of nutrition and ecdysone. Journal of Insect Physiology 43: 513-19.

Fellowes, M.D.E. (1999) The relative fitness of Drosophila melanogaster (Diptera: Drosophilidae) that have successfully defended themselves against the parasitoid Asobara tabida (Hymenoptera: Braconidae). Journal of Evolutionary Biology 12: 123-8.

Fellowes, M.D.E., Kraaijeveld, A.R. and Godfray, H.C.J. (1999) Cross-resistance following artificial selection for increased defence against parasitoids in Drosophila melanogaster. Evolution 53: $966-72$.

Flanders, S.E. (1950) Regulation of ovulation and egg disposal in the parasitic Hymenoptera. Canadian Entomologist 82: 134-40.

Gentry, G.L. and Dyer, L.A. (2002) On the conditional nature of neotropical caterpillar defenses against their natural enemies. Ecology 83: 3108-19. 
Giron, D. and Casas, J. (2003) Lipogenesis in an adult parasitic wasp. Journal of Insect Physiology 49: 141-7.

Giron, D., Rivero, A., Mandon, N., Darrouzet, E. and Casas, J. (2002) The physiology of hostfeeding in parasitic wasps: implications for survival. Functional Ecology 16: 750-7.

Giron, D., Ross, K.G. and Strand, M.R. (2007) Presence of soldier larvae determines the outcome of competition in a polyembryonic wasp. Journal of Evolutionary Biology 20: 165-72.

Godfray, H.C.J. (1994) Parasitoids: Behavioral and Evolutionary Ecology. Princeton University Press, Princeton.

Godfray, H.C.J. (2000) Host resistance, parasitoid virulence, and population dynamics. In: Hochberg, M.E. and Ives, A.R. (eds.) Parasitoid Population Biology. Princeton University Press, Princeton, pp. 121-38.

Gordon, M.D., Dionne, M.S., Schneider, D.S. and Nusse, R. (2005) WntD is a feedback inhibitor of Dorsal/NF-kappaB in Drosophila development and immunity. Nature 437: 746-9.

Gwynn, D.M., Callaghan, A., Gorham, J., Walters, K.F.A. and Fellowes, M.D.E. (2005) Resistance is costly: trade-offs between immunity, fecundity and survival in the pea aphid. Proceedings of the Royal Society of London Series B Biological Science 272: 1803-8.

Hanski, I. and Saccheri, I. (2006) Molecular-level variation affects population growth in a butterfly metapopulation. Plos Biology 4: 719-26.

Harrison, J.F. and Fewell, J.H. (2002) Environmental and genetic influences on flight metabolic rate in the honey bee, Apis mellifera. Comparative Biochemistry \& Physiology A 133: 255-8.

Harvey, J.A. and Strand, M.R. (2002) The developmental strategies of endoparasitoid wasps vary with host feeding ecology. Ecology 83: 2439-51.

Heidel, A.J., Clarke, J.D., Antonovics, J. and Dong, X. (2004) Fitness costs of mutations affecting the systemic acquired resistance pathway in Arabidopsis thaliana. Genetics 168: 2197-206.

Heimpel, G.E. and Collier, T.R. (1996) The evolution of host-feeding behaviour in insect parasitoids. Biological Reviews 71: 373-400.

Heimpel, G.E., Rosenheim, J.A. and Kattari, D. (1997) Adult feeding and lifetime reproductive success in the parasitoid Aphytis melinus. Entomologia Experimentalis et Applicata 83: 305-15.

Heimpel, G.E., Mangel, M. and Rosenheim, J.A. (1998) Effects of time limitation and egg limitation on lifetime reproductive success of a parasitoid in the field. American Naturalist 152: 273-89.

Henter, H. and Via, S. (1995) The potential for coevolution in a host-parasitoid system: 1. Genetic-variation within an aphid population in susceptibility to a parasitic wasp. Evolution 49: 427-38.

Hillyer, J.F., Schmidt, S.L., Fuchs, J.F., Boyle, J.P. and Christensen, B.M. (2005) Age-associated mortality in immune challenged mosquitoes (Aedes aegypti) correlates with a decrease in haemocyte numbers. Cellular Microbiology 7: 39-51.

Hochberg, M.E. (1997) Hide or flight? The competitive evolution of concealment and encapsulation in host-parasitoid associations. Oikos 80: 342-52.

Hoffmann, J.A. (2003) The immune response of Drosophila. Nature 426: 33-8.

Hufbauer, R.A. (2002) Evidence for nonadaptive evolution of parasitoid virulence following a biological control introduction. Ecological Applications 12: 66-78.

Irving, P., Ubeda, J.-M., Doucet, D. et al. (2005) New insights into Drosophila larval haemocyte function through genome-wide analysis. Cellular Microbiology 7: 335-50.

Jervis, M.A. and Kidd, N.A.C. (1986) Host-feeding strategies in hymenopteran parasitoids. Biological Reviews 61: 395-434.

Jervis, M.A., Heimpel, G.E., Ferns, P.N., Harvey, J.A. and Kidd, N.A.C. (2001) Life-history strategies in parasitoid wasps: a comparative analysis of 'ovigeny'. Journal of Animal Ecology 70: $442-58$.

Kidd, N.A.C. and Jervis, M.A. (1991a) Host-feeding and oviposition strategies of parasitoids in relation to host stage. Researches in Population Ecology 33: 13-28. 
Kidd, N.A.C. and Jervis, M.A. (1991b) Host-feeding and oviposition by parasitoids in relation to host stage: consequences for parasitoid-host population dynamics. Researches in Population Ecology 33: $87-9$.

Kraaijeveld, A.R. and Godfray, H.C.J. (1997) Trade-off between parasitoid resistance and larval competitive ability in Drosophila melanogaster. Nature 389: 278-80.

Kraaijeveld, A.R. and Godfray, H.C.J. (1999) Geographical patterns in the evolution of resistance and virulence in Drosophila and its parasitoids. American Naturalist 153: 61-74.

Kraaijeveld, A.R., van Alphen, J.J. and Godfray, H.C.J. (1998) The coevolution of host resistance and parasitoid virulence. Parasitology 116: 29-45.

Křivan, V. (1997) Dynamical consequences of optimal host feeding on host-parasitoid population dynamics. Bulletin of Mathematical Biology 59: 809-31.

Lavine, M.D. and Strand, M.R. (2002) Insect hemocytes and their role in cellular immune responses. Insect Biochemistry \& Molecular Biology 32: 1237-42.

Lochmiller, R.L. and Deerenberg, C. (2000) Trade-offs in evolutionary immunology: just what is the cost of immunity? Oikos 88: 87-98.

Mackauer, M. and Sequeira, R. (1993) Patterns of development in insect parasites. In: Beckage, N.E., Thompson, S.N. and Federici, B.A. (eds.) Parasites and Pathogens of Insects. Academic Press, New York, pp. 1-23.

Mangel, M. (1989) Evolution of host selection in parasitoids: does the state of the parasitoid matter? American Naturalist 133: 688-703.

Matarese, G. and La Cava, A. (2004) The intricate interface between the immune system and metabolism. Trends in Immunology 25: 193-200.

Mayhew, P.J. and Blackburn, T.M. (1999) Does development mode organize life-history traits in the parasitoid Hymenoptera? Journal of Animal Ecology 68: 906-19.

Min, K.-J., Hogan, M.F., Tatar, M. and O'Brian, D.M. (2006) Resource allocation to reproduction and soma in Drosophila: a stable isotope analysis of carbon from dietary sugar. Journal of Insect Physiology 52: 763-70.

Mondy, N., Corio-Costet, M.F., Bodin, A., Mandon, N., Vannier, F. and Monge, J.P. (2006) Importance of sterols acquired through host-feeding in synovigenic parasitoid oogenesis. Journal of Insect Physiology 52: 897-904.

Murdoch, W.W., Briggs, C.L. and Nisbet, R.M. (2003) Consumer-Resource Dynamics. Princeton University Press, Princeton.

O’Brien, D.M., Fogel, M.L. and Boggs, C.L. (2002) Renewable and nonrenewable resources: amino acid turnover and allocation to reproduction in Lepidoptera. Proceedings of the National Academy of Sciences USA 99: 4413-18.

O’Brien, D.M., Boggs, C.L. and Fogel, M.L. (2004) Making eggs from nectar: the role of life history and dietary carbon turnover in butterfly reproductive resource allocation. Oikos 105: 279-91.

Oldham, S., Montagne, J., Radimerski, T., Thomas, G. and Hafen, E. (2000) Genetic and biochemical characterization of dTOR, the Drosophila homolog of the target of rapamycin. Genes \& Development 18: 2689-94.

Olson, D.M., Fadamiro, H., Lundgren, J.G. and Heimpel, G.E. (2000) Effects of sugar feeding on carbohydrate and lipid metabolism in a parasitoid wasp. Physiological Entomology 25: 17-26.

Papaj, D.R. (2000) Ovarian dynamics and host use. Annual Review of Entomology 45: 423-48.

Pennacchio, F. and Strand, M.R. (2006) Evolution of developmental strategies in parasitic Hymenoptera. Annual Review of Entomology 51: 233-58.

Pennacchio, F., Digilio, M.C. and Tremblay, E. (1995) Biochemical and metabolic alterations in Acyrthosiphon pisum parasitized by Aphidius ervi. Archives of Insect Biochemistry \& Physiology 30: 351-67.

Price, P.W. (1974) Strategies for egg production. Evolution 28: 76-84.

Rahbé, Y., Digilio, M.C., Febvay, G., Guillaud, J., Fanti, P. and Pennacchio, F. (2002) Metabolic and symbiotic interactions in amino acid pools of the pea aphid, Acyrthosiphon pisum, parasitized by the braconid Aphidius ervi. Journal of Insect Physiology 48: 507-16. 
Raubenheimer, D. and Simpson, S.J. (1999) Integrating nutrition: a geometrical approach. Entomologia Experimentalis et Applicata 91: 67-82.

Rivero, A. and Casas, J. (1999) Incorporating physiology into parasitoid behavioural ecology: the allocation of nutritional resources. Researches in Population Ecology 41: 39-45.

Rivero, A. and West, S.A. (2002) The physiological costs of being small in a parasitic wasp. Evolutionary Ecology Research 4: 407-20.

Rivero, A., Giron, D. and Casas, J. (2001) Lifetime allocation of juvenile and adult nutritional resources to egg production in a holometabolous insect. Proceedings of the Royal Society Series B Biological Science 268: 1231-7.

Rivers, D.B. and Denlinger, D.L. (1994) Redirection of metabolism in the flesh fly, Sarcophaga bullata, following envenomation by the ectoparasitoid, Nasonia vitripennis, and correlation of metabolic effects with the diapause status of the host. Journal of Insect Physiology 40: 20715.

Rolff, J. and Siva-Jothy, M.T. (2002) Copulation corrupts immunity: a mechanism for the cost of mating in insects. Proceedings of the National Academy of Sciences USA 99: 9916-18.

Rolff, J. and Siva-Jothy, M.T. (2003) Invertebrate ecological immunity. Science 301: 472-5.

Rosenheim, J.A., Heimpel, G.E. and Mangel, M. (2000) Egg maturation, egg resorption and the costliness of transient egg limitation in insects. Proceedings of the Royal Society of London Series B Biological Science 267: 1565-73.

Sanders, A.E., Scarborough, C., Layen, S.J., Kraaijeveld, A.R. and Godfray, H.C.J. (2005) Evolutionary change in parasitoid resistance under crowded conditions in Drosophila melanogaster. Evolution 59: $1292-9$.

Salt, G. (1970) The Cellular Defence Reactions of Insects. Cambridge University Press, Cambridge.

Sasaki, A. and Godfray, H.C.J. (1999) A model for the coevolution of resistance and virulence in coupled host-parasitoid interactions. Proceedings of the Royal Society of London Series B Biological Science 266: 455-63.

Schmid-Hempel, P. and Ebert, D. (2003) On the evolutionary ecology of specific immune defence. Trends in Ecology \& Evolution 18: 27-32.

Scott, R.C., Schuldiner, O. and Neufeld, T.P. (2004) Role and regulation of starvation-induced autophagy in the Drosophila fat body. Developmental Cell 7: 167-78.

Shelby, K.S. and Webb, B.A. (1994) Polydnavirus infection inhibits synthesis of an insect plasma protein, arylphorin. Journal of General Virology 75: 2285-94.

Stacey, D.A. and Fellowes, M.D.E. (2002) Influence of temperature on pea aphid Acyrthosiphon pisum (Hemiptera: Aphididae) resistance to natural enemy attack. Bulletin of Entomological Research 92: 351-7.

Strand, M.R. (1986) The physiological interactions of parasitoids with their hosts and their influence on reproductive strategies. In: Waage, J. and Greathead, D. (eds.) Insect Parasitoids. Academic Press, London, pp. 97-136.

Strand, M.R. (2000) Life history variation and developmental constraints in parasitoids. In: Hochberg, M. and Ives, A.R. (eds.) Population Biology of Parasitoids. Princeton University Press, Princeton, pp. 139-62.

Strand, M.R. and Pech, L.L. (1995) Immunological compatibility in parasitoid-host relationships. Annual Review of Entomology 40: 31-56.

Thompson, S.N. (1982) Effects of parasitization by the insect parasite Hyposoter exiguae on growth, development, and physiology of its host Trichoplusia ni. Parasitology 84: 491-510.

Thompson, S.N. (1993) Redirection of host metabolism and effects on parasite nutrition. In: Beckage, N.E., Thompson, S.N. and Federici, B.A. (eds.) Parasites and Pathogens of Insects, vol. 1. Academic Press, New York, pp. 125-44.

Thompson, S.N. and Dahlman, D.L. (1998) Aberrant nutritional regulation of carbohydrate synthesis by parasitized Manduca sexta. Journal of Insect Physiology 44: 745-54.

Vogt, J.T., Appel, A.G. and West, M.S. (2000) Flight energetics and dispersal capability of the fire ant, Solenopsis invicta. Journal of Insect Physiology 46: 697-707. 
Vinson, S.B. (1990) Physiological interactions between the host genus Heliothis and its guild of parasitoids. Archives of Insect Biochemistry \& Physiology 13: 63-81.

Washburn, J.O., Wong, J.F. and Volkman, L.E. (2001) Comparative pathogenesis of Helicoverpa zea S nucleopolyhedrovirus in noctuid larvae. Journal of General Virology 82: 1777-84.

Watt, W.B. (1992) Eggs, enzymes, and evolution: natural genetic variants change insect fecundity. Proceedings of the National Academy of Sciences USA 89: 10608-12.

Weaver, R.J., Marris, G.C., Bell, H.A. and Edwards, J.P. (2001) Identity and mode of action of the host endocrine disrupters from the venom of parasitoid wasps. In: Edwards, J.P. and Weaver, R.J. (eds.) Endocrine Interactions of Insect Parasites and Pathogens. BIOS Scientific Publishers, Oxford, pp. 33-58.

Wertheim, B., Kraaijeveld, A.R., Schuster, E. et al. (2005) Genome wide expression in response to parasitoid attack in Drosophila. Genome Biology 6: R94.1-20.

$\mathrm{Wu}$, Q. and Brown, M.R. (2006) Signaling and function of insulin-like peptides in insects. Annual Review of Entomology 51: 1-24.

Yang, X. and Cox-Foster, D.L. (2005) Impact of an ectoparasite on the immunity and pathology of an invertebrate: evidence for host immunosuppression and viral amplification. Proceedings of the National Academy of Sciences USA 102: 7470-5.

Zera, A.J. and Zhao, Z. (2003) Life-history evolution and the microevolution of intermediary metabolism: activities of lipid-metabolizing enzymes in history morphs of a wing-dimophic cricket. Evolution 57: 586-96.

Zerofsky, M., Harel, E., Silverman, N. and Tatar, M. (2005) Aging of the innate immune response in Drosophila melanogaster. Aging Cell 4: 103-8.

Zhang, H., Stallock, J.P., Ng, J.C., Reinhard, C. and Neufeld, T.P. (2000) Regulation of cellular growth by the Drosophila target of rapamycin dTOR. Genes \& Development 14: 2112-724. 